\title{
De la métapopulation au voisinage : la génétique des populations en déséquilibre
}

\author{
D. COUVET, P. GOUYON *, F. KJELLBERG, Isabelle OLIVIERI (1), \\ D. POMENTE et G. VALDEYRON * \\ B2P-C.N.R.S., route de Mende, B.P. 5051, 34033 Montpellier Cedex \\ et $\left(^{*}\right)$ I.N.A.P.G., 16, rue Cl.-Bernard, 75231 Paris Cedex 05
}

\begin{abstract}
Résumé
La population est un concept pratique qui peut devenir un piège. En effet, diverses questions de génétique des populations ne peuvent pas être résolues si on se contente de les étudier à ce niveau.

Il est montré que l'intensité de la dispersion, dans la mesure où elle est génétiquement déterminée, n'est pas sélectionnée au niveau de la population au sens classique. Un modèle simple, étayé par des observations in-situ (sur Carduus), semble montrer qu'un ensemble plus vaste, la métapopulation, peut seul rendre compte des processus en jeu. De ce point de vue, l'espèce est considérée non plus comme composée de populations indépendantes mais de métapopulations où chaque population est régulièrement fondée par les autres et évolue ensuite sous l'action de phénomènes internes.

Ces processus internes se produisent-ils au moins tous au niveau de la population? Il semble que non pour certaines espèces au moins, peut-être une majorité, où chaque individu n'est potentiellement fécondé que par un échantillon non représentatif de l'ensemble. Ce problème avait déjà conduit WrIGHT à formuler le concept de voisinage. Chez le Thym, l'intégration des 3 niveaux (Métapopulation, Population et Voisinage) permet d'expliquer des phénomènes (taux très élevés de femelles) qui restaient incompréhensibles tant qu'on cherchait à les décrire par des modèles fondés sur la seule idée de population.
\end{abstract}

Mots clés : Structure des populations-dispersion-stérilité mâle-métapopulation-paysage.

\section{Summary}

From metapopulation to neighbourhood: genetics of unbalanced populations

The concept of population is very useful but can sometimes lead to dead ends. Indeed, various questions in population genetics cannot be solved if studied at this level.

It is shown that the intensity of dispersion, as far as it is genetically determined, does not respond to selection at the level of the population in its usual sens. A simple theorical

(1) Adresse actuelle : I.N.R.A., Domaine de Melgueil, 34130 Maugio. 
model in relation with in-situ observations (on Carduus), seems to show that à wider set, the metapopulation (GILL), is necessary to account for the processes concerned. From this viewpoint, instead of considering species as sets of independent populations, it is proposed to consider them as sets of metapopulations where individual populations are regulary founded by the others and then evolve under internal pressures.

Are these internal pressures acting at the very population level ? It does not seem so, at least for some, and perhaps for most species, since each individual is likely to mate with a subset which is not representative of the whole. This question has led WRIGHT to formulate the neighbourhood concept. In Thyme, the simultaneous integration of the emerging properties of the 3 levels (Metapopulation, Population and Neighbourhood) allows one to explain a phenomenon (very high proportions of females) which remained incomprehensible as long as one tried to describe it using only the population level.

Key words : Population structure-dispersal-male sterility-metapopulation-landscape.

\section{Introduction}

Les modèles classiques de génétique des populations sont fondés sur l'hypothèse selon laquelle la population est l'unité de microévolution. Il est ainsi possible d'étudier le maintien d'un polymorphisme à l'échelle de la population : au mieux, un facteur de migration à partir d'autres populations est introduit dans ces modèles. On est alors amené à étudier des situations à l'équilibre, dans lesquelles on détermine si un caractère donné peut ou non être maintenu (éventuellement à l'état polymorphe) lorsqu'il est soumis à des pressions de sélection, constantes ou variables, mais toujours définies à l'échelle de la population.

$\mathrm{Si}$ un caractère donné ne peut être maintenu à l'état polymorphe quelle que soit la population, on en déduit qu'il doit se fixer à plus ou moins long terme dans l'espèce; si l'on continue d'observer un polymorphisme, c'est que les populations n'ont pas encore atteint leur état d'équilibre. La recherche systématique d'un état d'équilibre au sein de la population est criticable, comme le fait remarquer MAYNARDSMITH (1978, p. 6) : " my own insight into the field may have been obscured by an obsession, which I share with most population biologists, with equilibrium situations $»$.

Dans cet article, nous voudrions montrer qu'il existe une échelle, différente de celle de la population, à laquelle un caractère donné peut être maintenu de façon stable à l'état polymorphe, alors que la direction de la sélection est la même quelle que soit la population.

\section{La dispersion chez les plantes : la notion de métapopulation}

Chez certaines espèces végétales, surtout des espèces colonisatrices, chaque individu peut produire simultanément 2 types de graines (Ollvieri \& Berger, 1985). Ce dimorphisme est souvent lié à une distance de dispersion différente selon la morphe considérée. Ainsi, chez Carduus tenuiflorus et Carduus pycnocephalus (Astéracées), chaque plante produit des akènes dispersables par le vent grâce au fait qu'ils portent une aigrette, et des akènes sans aigrette non dispersés. Ce dimorphisme des semences, 
aisément reconnaissable, permet de fournir une estimation de la capacité à disperser d'un individu - ou d'un ensemble d'individus - en mesurant la fréquence des akènes à aigrette. L'héritabilité de cette fréquence n'a pas été mesurée. Cependant, l'existence d'une composante génétique est très probable, dans la mesure où l'on observe (Olivieri, données non publiées) des différences systématiques, allant toujours dans le même sens, entre espèces différentes présentes dans un même site. Par exemple, il n'est pas rare d'observer 5 ou 6 akènes sans aigrettes dans chaque capitule de Carduus tenuiflorus, alors que ce nombre dépasse rarement trois ou quatre chez $C$. pycnocephalus. Les 2 espèces produisant chacune une trentaine d'akènes par capitule, le taux de dispersion se trouve ainsi systématiquement plus élevé chez $C$. pycnocephalus, si l'on considère une même population. On n'a jamais trouvé, que ce soit en études de descendances ou en populations naturelles, de plante ne présentant qu'un types de semences.

La fréquence de graines à aigrette a été mesurée en Californie, en 1983, dans 3 populations de $C$. tenuiflorus et 4 populations de $C$. pycnocephalus (OLIVIERI $\&$ GouYon, 1985). Cette fréquence varie en moyenne de 77 à 88 p. 100 (50 à 92 p. 100 si l'on prend en compte les individus extrêmes).

On observe que le pourcentage d'akènes à aigrette produits par individu est significativement plus grand dans les populations perturbées (bords de routes) que dans les populations plus stables (champs et forêts). Si l'on prend en compte lâge - estimé - de ces populations, on remarque que la capacité de dispersion des individus diminue quand l'âge de la population où ils sont présents augmente.

Ce résultat semble a priori surprenant. Chez une espèce colonisatrice, une population est l'objet d'un cycle plus ou moins rapide de colonisation comprenant 3 phases : colonisation, équilibre (c'est-à-dire densité maximale) puis extinction. Par conséquent une population occupe un milieu récemment colonisé d'autant plus vite qu'elle y laisse plus de descendants, c'est-à-dire qu'elle disperse moins de graines. Inversement, les individus d'une population en voie d'extinction devraient produire plus de graines dispersées, puisque les descendances laissées sur place ont peu de chances de s'y établir. Nous attendrions donc une augmentation de l'intensité de dispersion avec l'âge de la population si celle-ci était adoptée à son milieu. Le résultat - contraire semble donc un cas de sélection «disadaptative».

La génétique des populations permet, en fait, d'expliquer, au moins partiellement, cette contradiction (OLjvieri \& GouYon, 1985). En effet, si l'on considère la population comme unité d'évolution, les individus qui la constituent laissent d'autant moins de descendants sur place qu'ils en envoient plus au dehors. Dans une population donnée, tout se passe comme si le fait de migrer était létal pour une graine. Par cet effet de "sélection naturelle », il y a donc, dans un site donné, diminution de l'intensité de dispersion au cours des générations. L'équilibre au niveau du site semble n'être atteint que lorsque la capacité à disperser est devenue nulle. Or, aucune population n'est éternelle; par suite, si toutes les populations d'une espèce telle que $C$. pycnocephalus ou $C$. tenuiflorus évoluaient durant suffisamment de temps pour perdre totalement leur aptitude à la dispersion, aucune de ces populations ne laisserait de descendance lors de son extinction. Les espèces que nous observons doivent donc se trouver dans des conditions telles que les populations ne se maintiennent pas assez longtemps pour que l'espèce puisse perdre sa capacité à disperser. En effet, Olivieri \& GouYon (1985) ont montré que le bénéfice de la fondation d'une nouvelle population ne compensait pas le coût associé aux aléas de la dispersion. En 
revanche, la contre-sélection exercée sur les non-migrants au moment de l'extinction de la population s'avère être le facteur essentiel du maintien de l'aptitude à disperser. Cette sélection s'exerce non pas à l'échelle de la population (celle-ci s'éteint que les individus dispersent ou non), mais à l'échelle de la métapopulation (GILL, 1978, "constellation de populations discrètes, conspécifiques, représentant des unités de reproduction »), définie comme un ensemble de populations se fondant les unes à partir des autres, et évoluant indépendamment après la période de colonisation. Chaque nouvelle population est fondée à partir du «pool» de graines dispersées produites par la métapopulation. Les individus ayant une capacité de dispersion élevée ont une plus grande probabilité de coloniser un nouveau milieu via leurs descendants. Lors de la fondation d'une nouvelle population, il y a de ce fait sélection en faveur de la capacité de dispersion. Ainsi, à l'échelle de la métapopulation, une stratégie mixte peut être maintenue, alors que chaque population évolue vers une stratégie pure par une diminution de l'intensité de migration. Une population doit ici être définie comme un ensemble d'individus évoluant sous l'action de forces essentiellement internes (adaptations à des contraintes locales, dérive, etc.). Notamment le nombre de migrants arrivant à chaque génération est négligeable par rapport au nombre de descendants laissés par la population en place. Même si des individus à capacité de dispersion élevée immigrent à chaque génération dans une population donnée, ils ne compen. seront pas la contre-sélection de la capacité de dispersion qui a opéré sur l'ensemble des descendants des individus de la population. L'influence des migrants n'a lieu que si le nombre d'individus issus d'individus autochtones est quasiment nul, c'est-à-dire au moment de la fondation.

C'est l'ensemble de ces populations qui échangent des gènes entre elles (par l'intermédiaire des migrants) qui a été défini comme une métapopulation (GILL, 1978). Cet échange n'est quantitativement important que lors de la fondation d'une nouvelle population. Remarquons que cette métapopulation a pour «habitat " une unité écologique correspondant au "paysage» (Forman \& GodRon, 1981 ; Godron \& ForMAN, 1983 ; MERriam, 1984), c'est-à-dire un ensemble de sites présentant divers stades de succession écologique et dont la géographie autorise des échanges géniques limités mais existant d'un site à l'autre.

A l'échelle de la métapopulation, il existe ainsi 2 pressions de sélection antagonistes. On peut déterminer une intensité de dispersion évolutivement stable (celle qui sera favorisée) en fonction de la durée moyenne de vie d'une population. Si on suppose que cette durée moyenne de vie varie dans le temps (changements dans la métapopulation) ou dans l'espace (hétérogénéité du paysage), on obtient à l'état stable, un polymorphisme (Olivieri \& GouYON, 1985), c'est-à-dire le maintien de deux stratégies, mixtes toutes deux, pour la dispersion chez ces espèces colonisatrices.

Chez les espèces présentant un dimorphisme de semences pour la dispersion, on observe généralement que les graines non dispersées sont plus dormantes que les graines dispersées (Olivieri \& Berger, 1985). La dormance pourrait constituer un moyen de dispersion dans le temps, et elle l'est assurément pour certaines espèces. La colonisation de milieux nouvellement ouverts à partir de graines dormantes n'est certainement pas chose fréquente pour les espèces étudiées car on ne pourrait alors pas expliquer le fort taux de semences à aigrettes dans les jeunes populations.

Notons l'analogie et la différence entre notre approche et le concept de sélection de groupe, même nuancé. Chez Panicum maximum, espèce apomictique facultative, Pernes (1975 a, 1975 b) a montré que l'évolution interne des populations tend à 
faire disparaître la sexualité. Les probabilités d'extinction des populations, dans les milieux variables temporellement, augmentent d'autant plus que le taux de sexualité faible réduit les potentiels de variabilité. Un niveau optimum de sexualité résulte du fait que la décroissance interne de la sexualité a pour conséquence l'élimination (externe) des populations trop peu sexuées, lesquelles sont renouvelées par les migrants issus de populations dont la sexualité n'est pas encore descendue en dessous des seuils dangereux. La disparition du groupe (de la population) est le résultat de la sélection individuelle au sein du groupe. Dans notre approche, la disparition du groupe n'est aucunement reliée à sa structure génétique. Le groupe disparaît parce que les diverses espèces se remplacent au cours d'une succession.

\section{La gynodioecie : du voisinage à la métapopulation}

Thymus vulgaris est une espèce gynodioique, c'est-à-dire qu'il y existe un polymorphisme génétique du sexe : dans chaque population on trouve des femelles et des hermaphrodites. Le déterminisme génétique de ces deux formes sexuelles est probablement nucléocytoplasmique ; certaines informations cytoplasmiques déterminent la stérilité-mâle et à chacune de ces informations correspondent un ou plusieurs gènes nucléaires de restauration de la fertilité mâle (HENRY, 1976).

Comme pour Carduus, l'absence d'équilibre dans une même population apparaît lorsqu'on étudie la fréquence de la stérilité mâle dans les populations de Thym. Le taux de femelles est très élevé dans les populations jeunes; il peut atteindre 95 p. 100. Ce taux diminue dans les populations âgées, et peut descendre jusqu'à 5 p. 100 (DommÉe et al., 1978 ; Gouyon et al., 1983; DommÉE et al., 1983).

Les calculs théoriques (Lewis, 1941 ; Delannay et al., 1981 ; Charlesworth, 1981) prévoient que lorsque la présence de mâle-stériles ne dépend que de la disjonction de gènes nucléaires, leur proportion à l'équilibre ne peut dépasser 50 p. 100 . Le fait que cette proportion varie avec l'âge de la population entraîne que les populations jeunes ne sont pas en équilibre. En outre, il a été observé que, dans de telles populations, il y a une très forte hérédité maternelle de la forme femelle (ou mâlestérile) : les femelles ne donnent pratiquement que des femelles (Couvet et al., 1985) : la stérilité mâle y obéit donc à un déterminisme génétique cytoplasmique plıtôt que nucléaire. Par ailleurs, les femelles produisent en général 2 à 4 fois plus de graines que les hermaphrodites. Ces 2 facteurs, transmission élevée du caractère «femelle » et supériorité des femelles, permettent d'expliquer les taux très élevés de femelles dans ces populations. L'avantage femelle est, au moins partiellement, expliqué par une redistribution des ressources attribuées chez les hermaphrodites à la fonction mâle. En outre, les résultats théoriques et expérimentaux permettent d'expliquer l'existence d'une forte hérédité maternelle dans les jeunes populations (CouvET et al., 1985, Henry, 1976). Pour ce faire, la population ne doit plus être considérée comme un tout homogène mais comme occupant une surface nettement supérieure à ce qui est exploré par les gamètes de chaque individu à chaque génération. Dans cette optique, les parents (et descendants) d'un individu donné se trouvent potentiellement dans une aire située autour de lui et de taille d'autant plus réduite que la distance de dispersion est plus faible. Cette surface a été appelée voisinage de l'individu (Wright, 1965 ; Levin \& Kerster, 1971 ; Crawford, 1984 ; Cahalan \& Gliddon, 
1984). Si le voisinage de chaque individu peut être considéré comme homogène, cette notion ne se substitue cependant pas à la notion de population parce que (1) il existe un voisinage par individu, (2) deux voisinages différents peuvent avoir beaucoup d'individus en commun et (3) deux individus A et B peuvent avoir dans leur voisinage un même individu $\mathbf{C}$ sans que $A$ soit dans le voisinage de $B$ (la relation "être dans le voisinage de " est réflexive et symétrique mais non transitive contrairement à «être dans la même population que »).

Les graines chez le Thym se dispersant peu, on peut s'attendre à observer, dans une population en cours de fondation, 2 types de situation : (1) des agglomérations de femelles quand l'hermaphrodite voisin pollinisant la fondatrice ne possède pas les gènes de restauration de son cytoplasme, (2) des agglomérations plutôt hermaphrodites, quand le fondateur était un hermaphrodite ou une femelle qui a été fécondée par un hermaphrodite possédant les gènes de restauration de son cytoplasme. Une population colonisatrice de Thym est donc un ensemble de voisinages (chacun étant relativement homogène quant à son cytoplasme), qui croissent d'autant plus vite que leur fertilité mâle n'est pas restaurée. Par conséquent, la sténilité-mâle se répand durant les stades colonisateurs parce que les individus fondateurs d'une population sont différents quant à leur cytogénotype, vraisemblablement du fait qu'ils sont issus de populations différentes et que de forts effets spatiaux existent dans le milieu qu'elles colonisent. Les échanges génétiques restreints chez le Thym (Gouron, 1982 ; MAZzoni \& GouYon, 1985) ne permettent aux gènes de restauration de parvenir aux cytoplasmes non restaurés qu'après un certain laps de temps.

\section{Conclusion : le déséquilibre des populations}

Le maintien des 2 traits étudiés, la dispersion des graines et la stérilité-mâle, ne peut être envisagé à l'échelle de la population, où ils sont défavorisés. Ils sont, en revanche, favorisés lors de la fondation d'une nouvelle population, soit parce qu'ils accroissent la probabilité de trouver un nouveau milieu (la dispersion), soit parce qu'ils augmentent la vitesse de colonisation de ce nouveau milieu (la stérilitémâle qui augmente la production en graines). Leur maintien ne peut être expliqué qu'à l'échelle de la métapopulation. La compréhension de l'évolution de tels traits passe par l'abandon de l'idée de population stable dans le temps et dans l'espace. L'espèce n'est plus, de ce point de vue, un ensemble de populations évoluant plus ou moins parallèlement, et dont la cohésion ne provient que d'une occasionnelle migration. L'espèce est constituée d'un ensemble de populations en perpétuel déséquilibre. La condition de stabilité de l'ensemble du système réside paradoxalement dans la disparition inéluctable de chacune de ces populations corrélativement à l'apparition de nouvelles. La métapopulation, concept permettant l'établissement d'un équilibre global à une échelle supérieure à celle de la population, nous semble donc un objet d'étude primordial aussi bien pour l'écologue (voir l'engouement actuel pour les travaux relatifs à la notion de paysage) que pour l'évolutionniste.

Reçu le 4 décembre 1984.

Accepté le 20 février 1985. 


\section{Références bibliographiques}

Cahalan C.M., Gliddon C., 1985. Neighbourhood sizes in Primula vulgaris. Heredity, $55,65-70$.

Charlesworth D., 1981. A further study of the problem of the maintenance of females in gynodioecious species. Heredity, 46, 27-39.

CRAWFORD T.J., 1984: The estimation of neighbourhood parameters for plant populations. Heredity, 52, 273-283.

Couvet D., Gouyon P.H., KJellberg F., Valdeyron G., 1985. La différenciation nucléocytoplasmique entre populations. C.R. Acad. Sci., Paris. Série III, t. 300, 665-668.

DelannaY X., Gouyon P.H., Valdeyron G., 1981. Mathematical study of the evolution of gynodioecy with cytoplasmic inheritance ander the effect of a nuclear restorer gene. Genetics, 99, 169-181.

Dommee B., Assound M.W., Valdeyron G., 1978. Natural selection and gynodioecy in Thymus vulgaris L. Bot. J. Linnean Soc., 77, 17-28.

Dommee B., Guillerm J.L., ValdeYron G., 1983. Régime de reproduction et hétérozygotie des populations de Thym, Thymus vulgaris $L$., dans une succession postculturale. C.R. Acad. Sci. Paris. Série III, t. 296, 111-114.

Forman R.T.T., Godron M., 1981. Patches and structural components for a landscape ecology. Bio. Science, 31, 733-739.

GiLL D.E., 1978. The metapopulation ecology of the red-spotted newt, Notophtalmus viridescens (Rafinesque). Ecological monographs, 48, 145-166.

Godron M., Forman R.T.T., 1983. Landscape modifications and changing ecological characteristics. In : MOONEY H.A., Godron M. (ed.), "Disturbance and Ecosystem ", (Ecol. studies, 44), 12-28. Springer Verlag Heidelberg.

GouYON P.H., 1982. Système de reproduction, dispersion et diversité; une approche de la Biologie des populations du thym. Thèse Doct. Sci., U.S.T.L., Montpellier.

Gouyon P.H., Lumaret R., Valdeyron G., Vernet Ph., 1983. Reproductive strategy and disturbance by man. In : MOONEY H.A., GODRon M., (éd.), «Disturbance and Ecosystems », (Ecol. studies, 44), 12-28. Springer Verlag Heidelberg.

Henry J.P. 1976. Contribution à l'étude du déterminisme génétique de la stérilité-mâle chez Thymus vulgaris L. D.E.A. Amélioration des plantes, Université d'Orsay.

LeVIN D.A., KeRSTER H.W., 1971. Neighborhood structure in plants under diverse reproductive methods. Am. Nat., 105, 345-354.

LEwIS D., 1941. Male-sterility in natural populations of hermaphrodite plants. New Phytol., 40, 56-63.

MaYnaRd-Smith J., 1978. The evolution of sexe. 222 pp., Cambridge University Press. Cambridge.

MaYNARD-Smith J., 1982. Evolution and the Theory of games. 224 pp., Cambridge University Press, Cambridge.

Mazzoni C., GouYon P.H., 1985. Horizontal structure of populations, migration, adaptation and chance. In : Jacquard P., Heim G., Antonovics J. (ed.), «Genetic Differenciation and Dispersal in Plants », Springer Verlag (A paraître).

Merriam G., 1984. Connectivity : A fundamental ecological characteristic of landscape pattern. In : BRANDT \& AGGER (ed.), «Methodology in landscape ecological research and planning, Vol. 1, theme 1 : Landscape ecological concepts », 5-16.

Olivieri I., Berger A., 1985. Seed dimorphism for dispersal : physiological, genetic and demographical aspects. In : JacQUaRD P., HeIm G., Antonovics J. (ed.), «Genetic Differenciation and Dispersal in Plants ». Springer Verlag (à paraître).

Olivieri I., GouYon P.H.. 1985. Seed dimorphism for dispersal : theory and observations. In : Woldendorp (ed.), "Phenotypic and Genotypic Variations Within and Between Plant Populations》 (A paraître).

Pernes J., 1975 a. Biologie et populations naturelles du Panicum maximum Jacq. Cah. O.R.S.T.O.M. (sér. Biol.), vol. X (2), 77-89.

Wright S., 1965. Isolation by distance. Genetics, 28, 114-138. 